\title{
On the solutions of a boundary value problem arising in free convection with prescribed heat flux
}

\author{
Mohamed AÏBOUdI And Bernard BRIGHI*
}

\begin{abstract}
For given $a \in \mathbb{R}, c<0$, we are concerned with the solution $f_{b}$ of the differential equation $f^{\prime \prime \prime}+f f^{\prime \prime}+\mathbf{g}\left(f^{\prime}\right)=0$, satisfying the initial conditions $f(0)=a, f^{\prime}(0)=b$, $f^{\prime \prime}(0)=c<0$, where $\mathbf{g}$ is some nonnegative subquadratic locally Lipschitz function. It is proven that there exists $b_{*}>0$ such that $f_{b}$ exists on $[0,+\infty)$ and is such that $f_{b}^{\prime}(t) \rightarrow 0$ as $t \rightarrow+\infty$, if and only if $b \geq b_{*}$. This allows to answer questions about existence, uniqueness and boundedness of solutions to a boundary value problem arising in fluid mechanics, and especially in boundary layer theory.
\end{abstract}

AMS 2000 Subject Classification: 34B15; 34C11; 76D10.

Key words and phrases: Boundary layer, similarity solution, third order nonlinear differential equation, boundary value problem, fluid mechanics.

\section{Introduction.}

We consider the similarity third order differential equation

$$
f^{\prime \prime \prime}+f f^{\prime \prime}+\mathbf{g}\left(f^{\prime}\right)=0
$$

on $[0,+\infty)$, with the boundary conditions

$$
\begin{aligned}
& f(0)=a, \\
& f^{\prime \prime}(0)=c<0, \\
& f^{\prime}(+\infty):=\lim _{t \rightarrow+\infty} f^{\prime}(t)=0,
\end{aligned}
$$

where the function $\mathbf{g}: \mathbb{R} \rightarrow \mathbb{R}$ is assumed to be locally Lipschitz.

\footnotetext{
${ }^{*}$ Corresponding author.
} 
This boundary value problem with $\mathbf{g}(x)=\beta x^{2}$ arises in fluid mechanics, when looking for similarity solutions in free convection boundary-layer flows adjacent to permeable surfaces in porous media. The initial condition (3) means that heat flux is prescribed on the surface. In other situations, the surface temperature is prescribed, and in this case, condition (3) has to be replaced by $f^{\prime}(0)=b>0$. See for example [9] and [10] for details on the derivation of these problems, in the context of the boundary layer theory.

For $\mathbf{g}(x)=\beta x^{2}$, mathematical analysis of the problem with prescribed surface temperature is done in [2], [3], [8], [11], [12] and [14]. See also [7] and [4] for general function g. With prescribed surface heat flux, see [5] and [15].

In this paper, we are interested in the boundary value problem (1)-(41), with $0 \leq$ $\mathbf{g}(x) \leq x^{2}$. The particular case where $\mathbf{g}(x)=\beta x^{2}$ with $0<\beta<1$ corresponds to a question, which was not solved in [5], and which has obtained an answer in [15]. The method used by J.-C. Tsai and C.-A. Wang is based on the fact that $\mathbf{g}$ is homogeneous of degree 2, and on the study of a plane vector field associated to the differential equation (11). Here, we propose to revisit this question in a direct way, and, as far as possible, to prove results with $\mathbf{g}$ such that $0 \leq \mathbf{g}(x) \leq x^{2}$. We will see that, under this hypothesis, we are able to get existence of solutions, but that we will need to assume that $\mathbf{g}(x)=\beta x^{2}$ with $0<\beta<1$ to get more precise results (as uniqueness of the bounded solution). However, we think that this latter assumption is not necessary. In fact, for the boundary value problem involving (1) and the boundary conditions corresponding to prescribed surface temperature, it is possible to prove that the bounded solution is unique, only by assuming that $0 \leq \mathbf{g}(x) \leq x^{2}$, see [4].

\section{Preliminary remarks.}

The method to solve the boundary value problem (11)-(41) is shooting. For that, let $f_{b}$ denote the solution of the initial value problem :

$$
\left(\mathcal{P}_{\mathbf{g} ; a, b, c}\right) \quad\left\{\begin{array}{l}
f^{\prime \prime \prime}+f f^{\prime \prime}+\mathbf{g}\left(f^{\prime}\right)=0, \\
f(0)=a, \\
f^{\prime}(0)=b, \\
f^{\prime \prime}(0)=c,
\end{array}\right.
$$

and let $\left[0, T_{b}\right)$ be the right maximal interval of existence of $f_{b}$. To obtain a solution of the boundary value problem (11)-(4) amounts to find a value of $b$ such that $T_{b}=+\infty$ and $f_{b}^{\prime}(t) \rightarrow 0$ as $t \rightarrow+\infty$.

The following useful identities are obtained, by multiplying equation (11) by $1, f_{b}$ and $t$ respectively, and integrating by parts. For all $t \in\left[0, T_{b}\right)$, we have :

$$
\begin{gathered}
f_{b}^{\prime \prime}(t)-c+f_{b}(t) f_{b}^{\prime}(t)-a b=\int_{0}^{t}\left(f_{b}^{\prime}(s)^{2}-\mathbf{g}\left(f_{b}^{\prime}(s)\right)\right) d s \\
f_{b}(t) f_{b}^{\prime \prime}(t)-a c-\frac{1}{2} f_{b}^{\prime}(t)^{2}+\frac{1}{2} b^{2}+f_{b}(t)^{2} f_{b}^{\prime}(t)-a^{2} b=\int_{0}^{t} f_{b}(s)\left(2 f_{b}^{\prime}(s)^{2}-\mathbf{g}\left(f_{b}^{\prime}(s)\right)\right) d s
\end{gathered}
$$


and

$$
t f_{b}^{\prime \prime}(t)-f_{b}^{\prime}(t)+b+t f_{b}(t) f_{b}^{\prime}(t)-\frac{1}{2} f_{b}(t)^{2}+\frac{1}{2} a^{2}=\int_{0}^{t} s\left(f_{b}^{\prime}(s)^{2}-\mathbf{g}\left(f_{b}^{\prime}(s)\right)\right) d s
$$

We now give some lemmas, that we will use in the next sections.

Lemma 2.1. - If $T_{b}<+\infty$, then $f_{b}^{\prime \prime}(t)$ and $f_{b}^{\prime}(t)$ are unbounded as $t \rightarrow T_{b}$.

Proof. - First, if $T_{b}$ is finite, then $\left|f_{b}(t)\right|+\left|f_{b}^{\prime}(t)\right|+\left|f_{b}^{\prime \prime}(t)\right|$ is unbounded as $t \rightarrow T_{b}$. Then, necessarily $\left|f_{b}^{\prime \prime}(t)\right|$ is unbounded as $t \rightarrow T_{b}$, and using (5) we see that $\left|f_{b}^{\prime}(t)\right|$ is also unbounded as $t \rightarrow T_{b}$.

Remark 2.2. - In general, $f_{b}$ has no reason to be unbounded ; for example $f$ : $t \mapsto \sqrt{1-t}$ is the solution of $\left(\mathcal{P}_{\mathbf{g} ; 1,-1 / 2,-1 / 4}\right)$, with $\mathbf{g}(x)=x^{2}\left(1-12 x^{3}\right)$, on the maximal interval $[0,1)$ and $f^{\prime \prime}(t) \rightarrow-\infty, f^{\prime}(t) \rightarrow-\infty$ and $f(t) \rightarrow 0$ as $t \rightarrow 1$.

Lemma 2.3. - If $\mathbf{g}(x)>0$ for $x \neq 0$ and if $c<0$, then for any $b \in \mathbb{R}$ we have $f_{b}^{\prime \prime}<0$ on $\left[0, T_{b}\right)$, i.e. $f_{b}$ is concave.

Proof. - This follows from the relation $\left(f^{\prime \prime} e^{F}\right)^{\prime}=-\mathbf{g}\left(f^{\prime}\right) e^{F}$, where $F$ is any primitive function of $f$ on $\left[0, T_{b}\right)$.

Remark 2.4. - It is possible to show, under the assumptions of the previous lemma, that if $T_{b}=+\infty$ then $f_{b}^{\prime}>0$. Indeed, on the contrary, there would exists $t_{0}>0$ such that $f_{b}$ and $f_{b}^{\prime}$ are negative on $\left(t_{0},+\infty\right)$ and therefore $f_{b}$ would be a negative concave subsolution of the Blasius equation (i.e. satisfying $\left.f^{\prime \prime \prime}+f f^{\prime \prime} \leq 0\right)$ on $\left(t_{0},+\infty\right)$, and using similar arguments as the ones in the proofs of Proposition 2.1 and 2.2 of [1], we would obtain a contradiction. See also [4].

Lemma 2.5. - Let us assume that $0<\mathbf{g}(x) \leq 2 x^{2}$ for $x \neq 0, a<0$ and $c<0$. If $b>0$ is large enough, then there exists $s_{b} \in\left(0, T_{b}\right)$ such that $f_{b}\left(s_{b}\right)=0$ and $f_{b}^{\prime}\left(s_{b}\right)>\frac{3 b}{4}$.

Proof. - Since $f_{b}^{\prime \prime}(0)=c<0$, we deduce from Lemma 2.3 that $f_{b}^{\prime \prime}<0$ on $\left[0, T_{b}\right)$. Let us assume that there exists $t \in\left(0, T_{b}\right)$ such that $f_{b}(t) \leq 0$ and $f_{b}^{\prime}(t)=\frac{3 b}{4}$. Then, using (6) , we have

$$
-a c+\frac{7 b^{2}}{32}-a^{2} b=-f_{b}(t) f_{b}^{\prime \prime}(t)-f_{b}(t)^{2} f_{b}^{\prime}(t)+\int_{0}^{t} f_{b}(s)\left(2 f_{b}^{\prime}(s)^{2}-\mathbf{g}\left(f_{b}^{\prime}(s)\right)\right) d s \leq 0
$$

and then $b$ is smaller than the positive root of the polynomial $7 X^{2}-32 a^{2} X-32 a c$. This completes the proof. 


\section{The solutions of $\left(\mathcal{P}_{\mathrm{g} ; a, b, c}\right)$ when $\mathrm{g}$ is nonnegative and subquadratic.}

In this section, we will assume that $c<0$ and that $\mathbf{g}: \mathbb{R} \rightarrow \mathbb{R}$ is locally Lipschitz and such that $0<\mathbf{g}(x) \leq x^{2}$ for all $x \neq 0$. Let us notice that, by continuity, we have $\mathbf{g}(0)=0$.

By Lemma 2.3 , the function $f_{b}$ is concave on $\left[0, T_{b}\right)$, for all $b \in \mathbb{R}$. We will distinguish the following two types of behavior.

- Type (I) : $f_{b}^{\prime} \geq 0$ on $\left[0, T_{b}\right)$.

- Type (II) : there exists $t_{0} \in\left[0, T_{b}\right)$ such that $f_{b}^{\prime}<0$ on $\left(t_{0}, T_{b}\right)$.

We then define the sets

$$
\mathcal{B}_{1}=\left\{b \in \mathbb{R} ; f_{b} \text { is of type }(\mathrm{I})\right\} \quad \text { and } \quad \mathcal{B}_{2}=\left\{b \in \mathbb{R} ; f_{b} \text { is of type (II) }\right\} .
$$

Clearly, we have $\mathcal{B}_{1} \cap \mathcal{B}_{2}=\emptyset$ and $\mathcal{B}_{1} \cup \mathcal{B}_{2}=\mathbb{R}$. Moreover, $\mathcal{B}_{1}$ is a closed set (and hence $\mathcal{B}_{2}$ is an open set). In fact, if $b_{n} \in \mathcal{B}_{1}$ is a sequence converging to some $b_{*} \in \mathbb{R}$, and if $t \in\left[0, T_{b_{*}}\right)$, then, from the lower semicontinuity of the mapping $b \mapsto T_{b}$, there exists $n_{0} \in \mathbb{N}$ such that for $n \geq n_{0}$ we have $T_{b_{n}}>t$. Now, the continuity of $(b, t) \mapsto f_{b}^{\prime}(t)$ (defined for $b \in \mathbb{R}$ and $t \in\left[0, T_{b}\right)$ ), allows to write

$$
f_{b_{*}}(t)=\lim _{n \rightarrow+\infty} f_{b_{n}}(t) \geq 0
$$

and thus $b_{*} \in \mathcal{B}_{1}$.

On the other hand, it is clear that $(-\infty, 0] \subset \mathcal{B}_{2}$, or equivalently that $\mathcal{B}_{1} \subset(0,+\infty)$. A priori, nothing indicates that $\mathcal{B}_{1} \neq \emptyset$.

Remark 3.1. - It follows from Remark 2.4, that, if $b \in \mathcal{B}_{2}$, then $T_{b}<+\infty$.

The following result gives informations about $f_{b}$ for $b \in \mathcal{B}_{1}$.

Proposition 3.2. - If $b \in \mathcal{B}_{1}$, then $T_{b}=+\infty, f_{b}^{\prime}>0, f_{b}^{\prime}(t) \rightarrow 0$ as $t \rightarrow+\infty$, and there exists $t_{0} \geq 0$ such that $f_{b}(t)>0$ for all $t>t_{0}$. If, in addition, $f_{b}$ is bounded, then there exists a positive constant $A_{b}$ such that :

$$
\begin{aligned}
& f_{b}(t)=\mu_{b}-A_{b} e^{-\mu_{b} t(1+o(1))} \\
& f_{b}^{\prime}(t) \sim \mu_{b}\left(\mu_{b}-f_{b}(t)\right) \quad \text { and } \quad f_{b}^{\prime \prime}(t) \sim-\mu_{b} f_{b}^{\prime}(t)
\end{aligned}
$$

as $t \rightarrow+\infty$, where $\mu_{b}>0$ is the limit of $f_{b}(t)$ as $t \rightarrow+\infty$.

Proof. - Let $b \in \mathcal{B}_{1}$. Since $f_{b}^{\prime \prime}<0$ on $\left[0, T_{b}\right)$, then $f_{b}^{\prime}(t)$ has a nonnegative limit $\ell$ as $t \rightarrow T_{b}$ and thanks to Lemma 2.1, it follows that $T_{b}=+\infty$. Now, we claim that $\ell=01$.

\footnotetext{
${ }^{1}$ See Lemma 3 of [7] for a general proof of the fact that $\mathbf{g}(\ell)=0$.
} 
In fact, if $\ell>0$, then we have $f_{b}^{\prime}(t) \sim \ell$ and $f_{b}(t) \sim \ell t$ as $t \rightarrow+\infty$. Using (5) , since $\mathbf{g}(l)>0$, we get

$$
\begin{aligned}
f_{b}^{\prime \prime}(t)-c-a b & =-f_{b}(t) f_{b}^{\prime}(t)+\int_{0}^{t} f_{b}^{\prime}(s)^{2} d s-\int_{0}^{t} \mathbf{g}\left(f_{b}^{\prime}(s)\right) d s \\
& =-\ell^{2} t(1+\mathrm{o}(1))+\ell^{2} t(1+\mathrm{o}(1))-\mathbf{g}(\ell) t(1+\mathrm{o}(1)) \\
& =-\mathbf{g}(\ell) t+\mathrm{o}(t)
\end{aligned}
$$

which implies that $f_{b}^{\prime \prime}(t) \rightarrow-\infty$ as $t \rightarrow+\infty$. This is a contradiction with the fact that $f_{b}^{\prime}(t)$ has a finite limit as $t \rightarrow+\infty$. Therefore, $\ell=0$, and the positivity of $f_{b}^{\prime}$ then follows from the fact that $f_{b}^{\prime \prime}<0$.

Now, if $a \geq 0$ then $f_{b}$ is positive, and if $a<0$ and $f_{b}(t)<0$ for all $t \geq 0$ then we have

$$
\forall t \geq 0, \quad f_{b}^{\prime \prime \prime}=-f_{b} f_{b}^{\prime \prime}-\mathbf{g}\left(f_{b}^{\prime}\right)<0
$$

whence we deduce that $f_{b}^{\prime}$ is concave. This contradicts the fact that $f_{b}^{\prime}$ is positive and tends to 0 at infinity. Therefore, there exists $t_{0} \geq 0$ such that $f_{b}(t)>0$ for all $t>t_{0}$.

Finally, if $f_{b}$ is bounded, then we can integrate (11) between $t$ and $+\infty$; this gives :

$$
f_{b}^{\prime \prime}(t)+f_{b}(t) f_{b}^{\prime}(t)=\int_{t}^{+\infty}\left(\mathbf{g}\left(f_{b}^{\prime}(s)\right)-f_{b}^{\prime}(s)^{2}\right) d s
$$

Together with the assumptions about $\mathbf{g}$ and the fact that $f_{b}^{\prime}$ is positive and decreasing, we get :

$$
0 \geq \frac{f_{b}^{\prime \prime}(t)}{f_{b}^{\prime}(t)}+f_{b}(t) \geq-\frac{1}{f_{b}^{\prime}(t)} \int_{t}^{+\infty} f_{b}^{\prime}(s)^{2} d s \geq-\int_{t}^{+\infty} f_{b}^{\prime}(s) d s=f_{b}(t)-\mu_{b} .
$$

We immediatly deduce the second relation of (9) ; the first one follows from the L'Hôpital's rule, and (8) by an integration.

Proposition 3.3. - Let $\delta \in(0,1)$ and let us assume that $0<\mathbf{g}(x) \leq(1-\delta) x^{2}$ for all $x \neq 0$. There exists $b_{0}>0$ such that $\left[b_{0},+\infty\right) \subset \mathcal{B}_{1}$.

Proof. - As previously, let $f_{b}$ denote the solution of the initial value problem $\left(\mathcal{P}_{\mathbf{g} ; a, b, c}\right)$. Taking into account Lemma 2.5 and Proposition 3.2, we deduce that, for $b$ large enough, there exists $t_{b} \in\left(0, T_{b}\right)$ such that $f^{\prime}\left(t_{b}\right)=\frac{b}{2}$ and $f_{b}\left(t_{b}\right) \geq 0$. Using (5) and (7), we can write :

$$
\begin{array}{r}
t_{b} f_{b}^{\prime \prime}\left(t_{b}\right)+t_{b} f_{b}\left(t_{b}\right) f_{b}^{\prime}\left(t_{b}\right)-(c+a b) t_{b}=\int_{0}^{t_{b}} t_{b}\left(f_{b}^{\prime}(s)^{2}-\mathbf{g}\left(f_{b}^{\prime}(s)\right)\right) d s \\
t_{b} f_{b}^{\prime \prime}\left(t_{b}\right)+\frac{b}{2}+t_{b} f_{b}\left(t_{b}\right) f_{b}^{\prime}\left(t_{b}\right)-\frac{1}{2} f_{b}\left(t_{b}\right)^{2}+\frac{1}{2} a^{2}=\int_{0}^{t_{b}} s\left(f_{b}^{\prime}(s)^{2}-\mathbf{g}\left(f_{b}^{\prime}(s)\right)\right) d s
\end{array}
$$


By substraction, we obtain

$$
-(c+a b) t_{b}-\frac{b}{2}+\frac{1}{2} f_{b}\left(t_{b}\right)^{2}-\frac{1}{2} a^{2}=\int_{0}^{t_{b}}\left(t_{b}-s\right)\left(f_{b}^{\prime}(s)^{2}-\mathbf{g}\left(f_{b}^{\prime}(s)\right)\right) d s \geq 0 .
$$

Whence, together with the fact that $0 \leq f_{b}\left(t_{b}\right) \leq b t_{b}+a$, we get

$$
a^{2}+b+2(c+a b) t_{b} \leq f_{b}\left(t_{b}\right)^{2} \leq\left(b t_{b}+a\right)^{2}
$$

and hence $b^{2} t_{b}^{2}-2 c t_{b}-b \geq 0$. It follows that

$$
t_{b} \geq \frac{c+\sqrt{c^{2}+b^{3}}}{b^{2}}=\frac{b}{\sqrt{c^{2}+b^{3}}-c} .
$$

Then, if $f_{b}^{\prime}(t)=0$, we have $t>t_{b}$ and, using (5) and the assumptions about $\mathbf{g}$, we have

$$
\begin{aligned}
0>f_{b}^{\prime \prime}(t) & =c+a b+\int_{0}^{t}\left(f_{b}^{\prime}(s)^{2}-\mathbf{g}\left(f_{b}^{\prime}(s)\right)\right) d s \\
& \geq c+a b+\delta \int_{0}^{t} f_{b}^{\prime}(s)^{2} d s \geq c+a b+\delta \int_{0}^{t_{b}} f_{b}^{\prime}(s)^{2} d s \\
& \geq c+a b+\frac{\delta}{4} t_{b} b^{2} \geq c+a b+\frac{\delta}{4} \frac{b^{3}}{\sqrt{c^{2}+b^{3}}-c} .
\end{aligned}
$$

It follows that, if $b$ is large enough, then $f_{b}^{\prime}$ does not vanish, and hence $b \in \mathcal{B}_{1}$.

Remark 3.4. - If we only suppose that $\mathbf{g}(x) \leq x^{2}$, then the previous result may not hold ; for example, if $\mathbf{g}(x)=x^{2}$ and $a \leq 0, c<0$, then $\mathcal{B}_{1}=\emptyset$. Indeed, if there exists $b \in \mathcal{B}_{1}$, then $b>0$, and identity (5) gives $f_{b}^{\prime \prime}(t)+f_{b}(t) f_{b}^{\prime}(t)=c+a b$ for all $t \in \mathbb{R}$. Integrating, we obtain :

$$
\forall t \geq 0, \quad f_{b}^{\prime}(t)+\frac{1}{2} f_{b}(t)^{2}=b+\frac{1}{2} a^{2}+(c+a b) t
$$

and we see that the right hand side tends to $-\infty$ as $t \rightarrow+\infty$, whereas the left one is positive. We have a contradiction.

Proposition 3.5. - If $b$ is a point of the boundary of $\mathcal{B}_{1}$, then $f_{b}$ is bounded.

PROOF. - Since $b$ is on the boundary of $\mathcal{B}_{1}=\mathbb{R} \backslash \mathcal{B}_{2}$, there exists a sequence of positive real numbers $b_{n} \in \mathcal{B}_{2}$ converging to $b$. Let us set $f_{n}=f_{b_{n}}$ and $T_{n}=T_{b_{n}}$. Since $b_{n} \in \mathcal{B}_{2}$, there exists $t_{n} \in\left(0, T_{n}\right)$ such that $f_{n}^{\prime}\left(t_{n}\right)=0$. 
First, we remark that $t_{n} \rightarrow+\infty$ as $n \rightarrow+\infty$. On the contrary, there would exist an increasing subsequence $t_{n_{k}}$ converging to some $t<+\infty$. Because of the lower semicontinuity of the mapping $b \mapsto T_{b}$, for $k$ large enough, we should have $T_{n_{k}}>t$ and we could write

$$
0=\lim _{k \rightarrow+\infty} f_{n_{k}}^{\prime}\left(t_{n_{k}}\right) \geq \lim _{k \rightarrow+\infty} f_{n_{k}}^{\prime}(t)=f_{b}^{\prime}(t)
$$

and get a contradiction.

Next, using (17) for $t=t_{n}$ yields

$$
b-\frac{1}{2}\left(f_{n}\left(t_{n}\right)^{2}-a^{2}\right)=-t_{n} f_{n}^{\prime \prime}(t)+\int_{0}^{t_{n}} s\left(f_{n}^{\prime}(s)^{2}-\mathbf{g}\left(f_{n}^{\prime}(s)\right) d s \geq 0\right.
$$

and hence $f_{n}\left(t_{n}\right)^{2} \leq 2 b+a^{2}$. Since $f_{n}^{\prime}$ is positive on $\left[0, t_{n}\right)$, we obtain

$$
\forall s \in\left[0, t_{n}\right], \quad f_{n}(s) \leq \sqrt{2 b+a^{2}} .
$$

To conclude, we fix $t \in[0,+\infty)$. For $n$ large enough, we have $t_{n}>t$ and

$$
f_{b}(t)=\lim _{n \rightarrow+\infty} f_{n}(t) \leq \sqrt{2 b+a^{2}}
$$

This completes the proof.

\section{The boundary value problem (1)-(4) when $\mathrm{g}(x)=\beta x^{2}$ with $0<\beta<1$.}

Here, we consider the case where $\mathbf{g}(x)=\beta x^{2}$ with $0<\beta<1$. Our main result is the following.

Theorem 4.1. - Let $a \in \mathbb{R}$ and $c<0$. If $\mathbf{g}(x)=\beta x^{2}$ with $0<\beta<1$, then there exists $b_{*}>0$ such that $\mathcal{B}_{1}=\left[b_{*},+\infty\right)$. Moreover, if $b>b_{*}$, then $f_{b}$ is unbounded.

Proof. - Taking into account Propositions 3.3 and 3.5, it is sufficient to prove that there is at most one $b>0$ such that $f_{b}$ is bounded.

First, let us assume that for some $b>0$, the function $f=f_{b}$ is bounded. Let $\mu>0$ be the limit of $f(t)$ as $t \rightarrow+\infty$. Since $f$ is concave and increasing, then we can define a function $v:(0,1] \rightarrow \mathbb{R}$, such that

$$
\forall t \geq 0, \quad v\left(\frac{1}{b^{2}} f^{\prime}(t)^{2}\right)=\frac{1}{\sqrt{b}} f(t)
$$

By setting $y=\frac{1}{b^{2}} f^{\prime}(t)^{2}$, we get

$$
f(t)=\sqrt{b} v(y), \quad f^{\prime}(t)=b \sqrt{y}, \quad f^{\prime \prime}(t)=\frac{b^{3 / 2}}{2 v^{\prime}(y)} \quad \text { and } \quad f^{\prime \prime \prime}(t)=-\frac{b^{2} v^{\prime \prime}(y) \sqrt{y}}{2 v^{\prime}(y)^{3}} .
$$


Then, using (11) we obtain

$$
\forall y \in(0,1], \quad v^{\prime \prime}(y)=\frac{v(y) v^{\prime}(y)^{2}}{\sqrt{y}}+2 \beta \sqrt{y} v^{\prime}(y)^{3} .
$$

Moreover $v$ is decreasing on $(0,1]$, and we have

$$
v(1)=\frac{a}{\sqrt{b}}, \quad v^{\prime}(1)=\frac{b^{3 / 2}}{2 c} \quad \text { and } \quad \lim _{y \rightarrow 0} v(y)=\frac{\mu}{\sqrt{b}} .
$$

In addition, using (9), it holds

$$
v^{\prime}(y) \sim-\frac{\sqrt{b}}{2 \mu \sqrt{y}} \quad \text { as } \quad y \rightarrow 0
$$

Now, let us assume that there are $b_{1}>b_{2}$ such that the functions $f_{1}=f_{b_{1}}$ and $f_{2}=f_{b_{2}}$ are bounded. For $i=1,2$, let $\mu_{i}$ be the limit of $f_{i}(t)$ as $t \rightarrow+\infty$. Let $v_{i}:(0,1] \rightarrow \mathbb{R}$ be the corresponding solutions of (111).

If $w=v_{1}-v_{2}$, then $w$ is defined on $(0,1]$ and we have

$$
w(1)=\frac{a}{\sqrt{b}_{1}}-\frac{a}{\sqrt{b}_{2}}, \quad w^{\prime}(1)=\frac{1}{2 c}\left(b_{1}^{3 / 2}-b_{2}^{3 / 2}\right)<0 \quad \text { and } \quad \lim _{y \rightarrow 0} w(y)=\frac{\mu_{1}}{\sqrt{b}_{1}}-\frac{\mu_{2}}{\sqrt{b}_{2}} .
$$

Moreover, $w$ cannot have neither positive maximum, or negative minimum in $(0,1)$. Indeed, if $x \in(0,1)$ is such that $w(x)>0, w^{\prime}(x)=0$, then using (11) we have

$$
w^{\prime \prime}(x)=\frac{v_{1}^{\prime}(x)^{2}}{\sqrt{x}} w(x)>0 .
$$

The same arguments show that $w$ has no negative minimum in $(0,1)$.

We now distinguish between the cases $a \leq 0$ and $a>0$.

- If $a \leq 0$, then $w(1) \geq 0$. Since $w$ cannot have a positive maximum in $(0,1)$ and $w^{\prime}(1)<0$, it follows that $w(0)>0$. Thus, $\frac{\mu_{1}}{\sqrt{b}_{1}}>\frac{\mu_{2}}{\sqrt{b}_{2}}$ and hence, using (13), we get

$$
w^{\prime}(y) \sim-\frac{1}{2 \sqrt{y}}\left(\frac{\sqrt{b}_{1}}{\mu_{1}}-\frac{\sqrt{b}_{2}}{\mu_{2}}\right) \quad \text { as } \quad y \rightarrow 0 .
$$

Therefore, $w^{\prime}(y) \rightarrow+\infty$ as $y \rightarrow 0$ and this gives a contradiction since, on the contrary, $w$ should have a positive maximum in $(0,1)$.

- If $a>0$, then $w(1)<0$. Using the fact that $w$ cannot have neither positive maximum, or negative minimum in $(0,1)$ and the same arguments as previously, we obtain that necessarily we have $w^{\prime} \leq 0$ and $w(1)<w(0) \leq 0$. 
Now, for $i=1,2$, let us set $V_{i}=\frac{1}{v_{i}^{\prime}}$ and $W=V_{1}-V_{2}$. First, thanks to (13), we have $W(y) \rightarrow 0$ as $y \rightarrow 0$. Next, using (11), we obtain

$$
\begin{aligned}
\forall y \in(0,1], \quad W^{\prime}(y) & =-\frac{v_{1}^{\prime \prime}(y)}{v_{1}^{\prime}(y)^{2}}+\frac{v_{2}^{\prime \prime}(y)}{v_{2}^{\prime}(y)^{2}}=-\frac{w(y)}{\sqrt{y}}-2 \beta \sqrt{y} w^{\prime}(y) \\
& \leq-\frac{w(y)}{\sqrt{y}}-2 w^{\prime}(y) \sqrt{y}=-2(\sqrt{y} w(y))^{\prime}
\end{aligned}
$$

Integrating between 0 and 1 , we get $W(1) \leq-2 w(1)$. Thus,

$$
2 c\left(\frac{1}{b_{1}^{3 / 2}}-\frac{1}{b_{2}^{3 / 2}}\right) \leq-2 a\left(\frac{1}{\sqrt{b_{1}}}-\frac{1}{\sqrt{b_{2}}}\right) \text {. }
$$

Hence

$$
c\left(\frac{1}{b_{1}}+\frac{1}{\sqrt{b_{1} b_{2}}}+\frac{1}{b_{2}}\right) \geq-a
$$

and

$$
\frac{c}{b_{1}}+a \geq-c\left(\frac{1}{\sqrt{b_{1} b_{2}}}+\frac{1}{b_{2}}\right)>0 .
$$

This is contradiction, since thanks to (15) written for $f_{1}$ and $t \rightarrow+\infty$ we must have $c+a b_{1}<0$.

The proof is complete.

Remark 4.2. - If $b>b_{*}$, then there exists a positive constant $A_{b}$ such that

$$
f_{b}(t) \sim A_{b} t^{\frac{1}{1+\beta}} \quad \text { as } t \rightarrow+\infty
$$

See [6] and [11].

Corollary 4.3. - Let $a \in \mathbb{R}$ and $c<0$. If $\mathbf{g}(x)=\beta x^{2}$ with $0<\beta<1$, then the boundary value problem (11)-(41) has exactly one bounded solution, and infinitely many unbounded solutions.

PROOF. - This follows immediatly from the previous theorem.

Corollary 4.4. - Let $a \in \mathbb{R}$ and $m \in\left(-1,-\frac{1}{2}\right)$. The boundary value problem

$$
\left\{\begin{array}{l}
f^{\prime \prime \prime}+(m+2) f f^{\prime \prime}-(2 m+1) f^{\prime 2}=0 \text { on }[0,+\infty) \\
f(0)=a, \quad f^{\prime \prime}(0)=-1, \quad f^{\prime}(t) \rightarrow 0 \text { as } t \rightarrow+\infty
\end{array}\right.
$$

has exactly one bounded solution, and infinitely many unbounded solutions. 
Proof. - By setting

$$
\frac{1}{\sqrt{m+2}} \tilde{f}(t)=f\left(\frac{t}{\sqrt{m+2}}\right)
$$

we see that $f$ is a solution of the boundary value problem (15) if and only if $\tilde{f}$ is a solution

of the boundary value problem (1)-(4) with $\mathbf{g}(x)=\beta x^{2}$ and $\beta=-\frac{2 m+1}{m+2}$. The proof then follows from Corollary 4.3 ,

\section{Conclusion.}

The result of Theorem 4.1 is obtained by using strongly the fact that $\mathbf{g}$ is homogeneous of degree 2, contrary to the results of sections 2 and 3, where only the subquadratic nature of $\mathbf{g}$ is used. Nevertheless, we conjecture that the uniqueness of the bounded solution should hold under the hypothesis $0 \leq \mathrm{g}(x) \leq x^{2}$. In fact, the boundary value problem

$$
\left\{\begin{array}{l}
f^{\prime \prime \prime}+f f^{\prime \prime}+\mathbf{g}\left(f^{\prime}\right)=0 \text { on }[0,+\infty) \\
f(0)=a, \quad f^{\prime}(0)=b>0, \quad f^{\prime}(t) \rightarrow 0 \text { as } t \rightarrow+\infty,
\end{array}\right.
$$

has at most one bounded concave solution, if $0 \leq \mathrm{g}(x) \leq x^{2}$, see [4], and this result is one of the reasons for which we hope that this conjecture holds.

On the other hand, let us notice that we recover the results of J.-C. Tsai and C.-A. Wang [15], in a totally different way, and perhaps more directly.

\section{References}

[1] Z. Belhachmi, B. Brighi and K. Taous, On the concave solutions of the Blasius equation, Acta Math. Univ. Comenian. 69 (2000), no. 2, 199-214.

[2] Z. Belhachmi, B. Brighi and K. Taous, On a family of differential equations for boundary layer approximations in porous media, European J. Appl. Math. 12 (2001), no. $4,513-528$.

[3] B. Brighi, On a similarity boundary layer equation, Z. Anal. Anwendungen 21 (2002), no. 4, 931-948.

[4] B. Brighi, On the differential equation $f^{\prime \prime \prime}+f f^{\prime \prime}+\mathbf{g}\left(f^{\prime}\right)=0$ and the associated boundary value problems, preprint.

[5] B. Brighi and J.-D. Hoernel, On similarity solutions for boundary layer flows with prescribed heat flux, Math. Methods Appl. Sci. 28 (2005), no. 4, 479-503.

[6] B. Brighi and J.-D. Hoernel, Asymptotic behavior of the unbounded solutions of some boundary layer equation, Arch. Math. (Basel) 85 (2005), no. 2, 161-166. 
[7] B. Brighi and J.-D. Hoernel, On a general similarity boundary layer equation, preprint (http://arxiv.org/abs/math/0601385).

[8] B. Brighi and T. Sari, Blowing-up coordinates for asimilarity boundary layer equation, Discrete Contin. Dyn. Syst. (Serie A) 12 (2005), no. 5, 929-948.

[9] M. A. Chaudhary, J.H. Merkin and I. Pop, Similarity solutions in free convection boundary-layer flows adjacent to vertical permeable surfaces in porous media: I prescribed surface temperature, European J. Mech. B Fluids 14 (1995), no. 2, 217 237.

[10] M. A. Chaudhary, J.H. Merkin and I. Pop, Similarity solutions in free convection boundary-layer flows adjacent to vertical permeable surfaces in porous media: II prescribed surface heat flux, Heat and Mass Transfer 30 (1995), 341-347.

[11] M. Guedda, Similarity solutions of differential equations for boundary layer approximations in porous media, J. Appl. Math. Phys. (ZAMP) 56 (2005), 749-762.

[12] J.-S. Guo and J.-C. Tsai, The structure of the solutions for a third order differential equation in boundary layer theory, Japan J. Indust. Appl. Math. 22 (2005), no. 3, 311-351.

[13] P. Hartmann, Ordinary Differential Equations. Wiley, New-York (1964).

[14] J.-C. Tsai, Similarity solutions for boundary layer flows with prescribed surface temperature, to appear in Appl. Math. Lett., available online 20 March 2007.

[15] J.-C. Tsai and C.-A. Wang, A note on similarity solutions for boundary layer flows with prescribed heat flux, to appear in Math. Methods Appl. Sci., available online 15 March 2007.

Mohamed Aïboudi

Université d'Oran (Es-Senia)

Département de Mathématiques

BP 1524

El Menouar

31000 Oran

Algeria

e-mail : m.aiboudi@yahoo.fr

Bernard Brighi

Université de HauteAlsace

Laboratoire de Mathématiques, Informatique et Applications

4 rue des frères Lumière

F-68093 Mulhouse cedex

France

e-mail : bernard.brighi@uha.fr 